\title{
APPLICATIONS OF NIELSEN THEORY TO DYNAMICS
}

\author{
BOJU JIANG \\ Department of Mathematics, Peking University \\ Beijing 100871, China \\ E-mail: jiangbj@sxx0.math.pku.edu.cn
}

\begin{abstract}
In this talk, we shall look at the application of Nielsen theory to certain questions concerning the "homotopy minimum" or "homotopy stability" of periodic orbits under deformations of the dynamical system. These applications are mainly to the dynamics of surface homeomorphisms, where the geometry and algebra involved are both accessible.
\end{abstract}

1. Introduction. Fixed point theory is a theory of mathematical equations. Many equations can be written in the standard form $x=f(x)$ for a suitable space $X$ and a map $f: X \rightarrow X$. A solution to such an equation is called a fixed point of the map $f$. The fixed point set of $f$ is the set Fix $f:=\{x \in X \mid x=f(x)\}$. We are concerned with the existence, properties, computation, etc. of the fixed points. In topology, we are more interested in the behavior of the fixed point set Fix $f$ under deformations of the map $f$. In other words, we study homotopy invariants relevant to the fixed point problem. A problem challenging enough to attract the most attention is to find the minimal number of fixed points for maps homotopic to a given map, i.e. to determine

$$
M F[f]:=\operatorname{Min}\{\# \operatorname{Fix} g \mid g \simeq f: X \rightarrow X\} .
$$

This is the main theme of the classical Nielsen fixed point theory.

In dynamics, the main concern is the behavior of the orbits of a map $f$, i.e. sets of the form $O_{f}(x):=\left\{f^{n}(x) \mid 1 \leq n<\infty\right\}$, among which the periodic ones play an important role. So the study of the fixed points of the iterates of a map $f$ (i.e. periodic points or periodic orbits of $f$ ) is in order. Homotopy stability refers to dynamical behavior that persists under deformation. For periodic orbits it means the presence of certain types of such for all maps in a homotopy class. One can also ask whether there is a map in the homotopy class that has only the forced complexity and no more, i.e. a minimal representative with respect to the dynamical behavior in question. See [Bo1] for an exposition.

1991 Mathematics Subject Classification: Primary 55M20; Secondary 57N05.

Partially supported by NSFC.

The paper is in final form and no version of it will be published elsewhere. 
In this talk, we shall look at the application of Nielsen theory to certain questions concerning the homotopy minimum of periodic orbits. These applications are mainly to the dynamics of surface homeomorphisms, where the geometry and algebra involved are both accessible.

The exposition is divided into four sections. To set the stage, Section 2 sketches the Nielsen theory of periodic orbits, emphasizing the Lefschetz numbers and the Lefschetz zeta function, rather than the Nielsen numbers. Section 3 deals with homeomorphisms of compact surfaces and punctured surfaces. The asymptotic Nielsen number is identified with the largest stretching factor in the Thurston canonical form. Minimal representatives in isotopy classes are discussed. Section 4 is devoted to orientation preserving homeomorphisms of the plane, or more precisely, of the punctured disk. Here braids come into play. After presenting the recipe for calculating the Lefschetz zeta function, the estimation of asymptotic invariants is considered. The linking and braiding of periodic orbits will be discussed in some detail. Section 5 focuses on the set of periods of a map. We shall consider two aspects concerning this set, namely the degree of fixed point freedom and the minimal set of periods.

2. Nielsen theory for periodic orbits. The connection between Nielsen fixed point theory and dynamics was first explored by Fuller in the pioneering work [Fu2]. We shall describe the mapping torus approach of extending the classical Nielsen fixed point theory to periodic orbits, as proposed in [J3]. See [Fr] and [GN] for other approaches.

We start with a review of the classical Lefschetz and Nielsen fixed point theorems and then turn to iterates of maps. Instead of counting periodic points of $f$ (i.e. fixed points of $f^{n}$ ), we count the periodic orbits of $f$ and introduce the notion of periodic orbit classes. This allows for a natural interpretation on the mapping torus $T_{f}$ of $f$, and thus leads to familiar algebraic machinery. Associated to matrix representations of the fundamental group $\pi_{1}\left(T_{f}\right)$, we introduce the notion of zeta functions of $f$. The zeta function is a formal power series that encodes periodic orbit information of all periods. On the other hand it is a rational function that is practically computable. The asymptotic growth rate of the Nielsen numbers is a homotopy lower bound to the topological entropy (an important measure of complexity in dynamics). Methods of its estimation are proposed.

2.1. The classical notions. Unless otherwise stated, we always assume that the space $X$ is a compact connected polyhedron.

A notion central to topological fixed point theory is the index of an isolated set of fixed points. It generalizes the notion of multiplicity for solutions of polynomial equations. See $[\mathrm{B}]$ or $[\mathrm{D}]$ for a modern treatment. The following Lefschetz Theorem is probably the best known and most useful fixed point theorem.

Lefschetz-Hopf TheOREm ([L], $[\mathrm{H}])$. The algebraic sum $L(f)$ of indices of all fixed points of $f$ is a homotopy invariant of $f$. It can be computed via homology

$$
L(f)=\sum_{q}(-1)^{q} \operatorname{trace}\left(f_{q}: H_{q}(X) \rightarrow H_{q}(X)\right) .
$$

Hence when $L(f) \neq 0$ every map homotopic to $f$ must have a fixed point. 
The invariant $L(f)$ is called the Lefschetz number of $f$. It is an algebraic count of fixed points, not the number of geometrically distinct fixed points.

ExAmple. For the torus $T^{2}$, the homology homomorphism $f_{1}: H_{1}\left(T^{2}\right) \rightarrow H_{1}\left(T^{2}\right)$ is characterized by a $2 \times 2$ integral matrix $A$. Then $L(f)=\operatorname{det}(I-A)$, where $I$ is the identity matrix.

Another theory emerged from a beautiful result for the torus.

Nielsen-Brouwer Theorem ([N1], [Br]). In the homotopy class of a map $f: T^{2} \rightarrow$ $T^{2}$, the minimal number of fixed points is exactly $|\operatorname{det}(I-A)|$.

Nielsen later developed his theory [N2] for homeomorphisms of oriented closed surfaces of genus $g>1$. The central notion is that of a fixed point class. A fixed point class of $f: X \rightarrow X$ is the projection of the fixed point set of a lifting $\tilde{f}: \widetilde{X} \rightarrow \widetilde{X}$ of $f$, where $\widetilde{X}$ is the universal covering of $X$. Alternatively, two fixed points are in the same class if and only if they can be joined by a path which is homotopic (relative to end points) to its own $f$-image. Each fixed point class $\mathbf{F}$ is an isolated subset of Fix $f$, hence its index $\operatorname{ind}(\mathbf{F}, f) \in \mathbb{Z}$ is defined. A fixed point class is called essential if its index is non-zero. The number of essential fixed point classes is called the Nielsen number $N(f)$ of $f$. Nielsen's theory was generalized to compact connected polyhedra by Wecken [W].

Nielsen-WeCken TheOrem ([N2], [W]). $N(f)$ is a homotopy invariant of $f$. Every map homotopic to $f$ must have at least $N(f)$ distinct fixed points. Hence $N(f) \leq M F[f]$.

This theorem shows $N(f)$ is a lower bound of the geometric count of fixed points. However, it does not provide an effective way to compute $N(f)$. Thus the problem of determining $M F[f]$ splits into two: the more algebraic one of computing $N(f)$, and the more geometric one of investigating the equality or difference between $N(f)$ and $M F[f]$. For modern treatments of Nielsen fixed point theory, see the books [B], [J1] and $[\mathrm{K}]$.

2.2. Periodic orbit class via the mapping torus. A fixed point $x$ of $f^{n}$ will be called an $n$-point of $f,\left\{x, f(x), \ldots, f^{n-1}(x)\right\}$ an $n$-orbit of $f$. It is called a primary $n$-orbit if it consists of $n$ distinct points, i.e. if $n$ is the least period of the periodic point $x$.

A fixed point class $\mathbf{F}^{n}$ of $f^{n}$ will be called an $n$-point class of $f$.

We shall look at periodic orbits of $f$ on the mapping torus. The mapping torus $T_{f}$ of $f: X \rightarrow X$ is the space obtained from $X \times \mathbb{R}_{+}$by identifying $(x, s+1)$ with $(f(x), s)$ for all $x \in X, s \in \mathbb{R}_{+}$, where $\mathbb{R}_{+}$stands for the real interval $[0, \infty)$. On $T_{f}$ there is a natural semi-flow ("sliding along the rays")

$$
\varphi: T_{f} \times \mathbb{R}_{+} \rightarrow T_{f}, \quad \varphi_{t}(x, s)=(x, s+t) \text { for all } t \geq 0,
$$

which is known as the "suspension semi-flow" of the map $f$ in dynamics. A point $x \in X$ and a positive number $\tau>0$ determine an orbit curve $\varphi_{(x, \tau)}:=\left\{\varphi_{t}(x)\right\}_{0 \leq t \leq \tau}$ in $T_{f}$. We may identify $X$ with the cross-section $X \times 0 \subset T_{f}$, then the map $f: X \rightarrow X$ is just the return map of the semi-flow $\varphi$.

Notation. Let $\Gamma$ be the fundamental group $\Gamma:=\pi_{1}\left(T_{f}\right)$ and let $\Gamma_{c}$ denote the set of conjugacy classes in $\Gamma$. We shall regard $\Gamma_{c}$ as the set of free homotopy classes of closed curves in $T_{f}$, so that it is independent of the base point of $T_{f}$. Let $\mathbb{Z} \Gamma$ be the integral 
group ring of $\Gamma$, and let $\mathbb{Z} \Gamma_{c}$ be the free abelian group with basis $\Gamma_{c}$. We use the bracket notation $\alpha \mapsto[\alpha]$ for both projections $\Gamma \rightarrow \Gamma_{c}$ and $\mathbb{Z} \Gamma \rightarrow \mathbb{Z} \Gamma_{c}$. The norm in $\mathbb{Z} \Gamma_{c}$ is defined by $\left\|\sum_{i} k_{i}\left[\gamma_{i}\right]\right\|:=\sum_{i}\left|k_{i}\right| \in \mathbb{Z}$ when the $\left[\gamma_{i}\right]$ 's in $\Gamma_{c}$ are all different.

Observe that $x \in$ Fix $f^{n}$ if and only if on the mapping torus $T_{f}$ the time- $n$ orbit curve $\varphi_{(x, n)}$ is a closed curve. We define $x, y \in$ Fix $f^{n}$ to be in the same $n$-orbit class if and only if the closed curves $\varphi_{(x, n)}$ and $\varphi_{(y, n)}$ are freely homotopic in $T_{f}$. Fix $f^{n}$ splits into a disjoint union of $n$-orbit classes. It turns out that each $n$-orbit class is an $f$-orbit of $n$-point classes.

Let $\mathbf{O}^{n}$ be an $n$-orbit class. Since for all $x \in \mathbf{O}^{n}$ the closed curves $\varphi_{(x, n)}$ are freely homotopic in $T_{f}$, they represent a well defined conjugacy class $\left[\varphi_{(x, n)}\right]$ in $\Gamma$. This conjugacy class will be called the coordinate of $\mathbf{O}^{n}$ in $\Gamma$, written

$$
\operatorname{cd}_{\Gamma}\left(\mathbf{O}^{n}\right)=\left[\varphi_{(x, n)}\right] \in \Gamma_{c} .
$$

Suppose $m$ is a proper factor of $n$ and $m<n$. When the $n$-orbit class $\mathbf{O}^{n}$ contains an $m$-orbit class $\mathbf{O}^{m}$ then $\operatorname{cd}_{\Gamma}\left(\mathbf{O}^{n}\right)$ is the $(n / m)$-th power of $\operatorname{cd}_{\Gamma}\left(\mathbf{O}^{m}\right)$ because, for $x \in \mathbf{O}^{m}$, the closed curve $\varphi_{(x, n)}$ is the closed curve $\varphi_{(x, m)}$ traced $n / m$ times. This motivates the definition that the $n$-orbit class $\mathbf{O}^{n}$ is reducible to period $m$ if $\operatorname{cd}_{\Gamma}\left(\mathbf{O}^{n}\right)$ has an $(n / m)$-th root, and that $\mathbf{O}^{n}$ is irreducible if $\operatorname{cd}_{\Gamma}\left(\mathbf{O}^{n}\right)$ is primary in the sense that it has no nontrivial root.

Every $n$-orbit class $\mathbf{O}^{n}$ is an isolated subset of Fix $f^{n}$. Its $i n d e x$ is $\operatorname{ind}\left(\mathbf{O}^{n}, f^{n}\right)$, the index of $\mathbf{O}^{n}$ with respect to $f^{n}$. An $n$-orbit class $\mathbf{O}^{n}$ is called essential if its index is non-zero.

For each natural number $n$, the (generalized) Lefschetz number (with respect to $\Gamma$ ) is defined as

$$
L_{\Gamma}\left(f^{n}\right):=\sum_{\mathbf{O}^{n}} \operatorname{ind}\left(\mathbf{O}^{n}, f^{n}\right) \cdot \operatorname{cd}_{\Gamma}\left(\mathbf{O}^{n}\right) \in \mathbb{Z} \Gamma_{c},
$$

the summation being over all $n$-orbit classes $\mathbf{O}^{n}$ of $f$.

The number of non-zero terms in $L_{\Gamma}\left(f^{n}\right)$ will be denoted $N_{\Gamma}\left(f^{n}\right)$, and called the $n$-orbit Nielsen number of $f$. It is the number of essential $n$-orbit classes, a lower bound for the number of $n$-orbits of $f$. The norm $\left\|L_{\Gamma}\left(f^{n}\right)\right\|$ is the sum of absolute values of the indices of all the (essential) $n$-orbit classes. It equals the sum of absolute values of the indices of all the (essential) $n$-point classes, because any two $n$-point classes contained in the same $n$-orbit class must have the same index. Hence $\left\|L_{\Gamma}\left(f^{n}\right)\right\| \geq N\left(f^{n}\right) \geq N_{\Gamma}\left(f^{n}\right)$.

Similarly define the irreducible Lefschetz number

$$
L I_{\Gamma}\left(f^{n}\right):=\sum_{\text {irreducible } \mathbf{O}^{n}} \operatorname{ind}\left(\mathbf{O}^{n}, f^{n}\right) \cdot \operatorname{cd}_{\Gamma}\left(\mathbf{O}^{n}\right) \in \mathbb{Z} \Gamma_{c},
$$

the summation being over all irreducible $n$-orbit classes $\mathbf{O}^{n}$ of $f$.

Let $N I_{\Gamma}\left(f^{n}\right)$ be the number of non-zero terms in $L I_{\Gamma}\left(f^{n}\right)$, called the irreducible $n$ orbit Nielsen number of $f$. It is the number of irreducible essential $n$-orbit classes, a lower bound for the number of primary $n$-orbits.

The basic invariance properties, such as the homotopy invariance and the commutativity property, are similar to that for fixed points (cf. [J1, §§I.4-5]). For example: 
Homotopy invariance. Suppose $f \simeq f^{\prime}: X \rightarrow X$ via a homotopy $\left\{f_{t}\right\}_{0 \leq t \leq 1}$. The homotopy gives rise to a homotopy equivalence $T_{f} \simeq T_{f^{\prime}}$ in a standard way. If we identify $\Gamma^{\prime}=\pi_{1}\left(T_{f^{\prime}}\right)$ with $\Gamma=\pi_{1}\left(T_{f}\right)$ via this homotopy equivalence, then $L_{\Gamma^{\prime}}\left(f^{\prime n}\right)=L_{\Gamma}\left(f^{n}\right)$ for all $n$, hence also $N_{\Gamma^{\prime}}\left(f^{\prime n}\right)=N_{\Gamma}\left(f^{n}\right), L I_{\Gamma^{\prime}}\left(f^{\prime n}\right)=L I_{\Gamma}\left(f^{n}\right)$ and $N I_{\Gamma^{\prime}}\left(f^{\prime n}\right)=N I_{\Gamma}\left(f^{n}\right)$.

REMARK. When $n=1, L_{\Gamma}(f)$ is, in spirit, the same as the classical invariant called the Reidemeister trace $([\mathrm{R}],[\mathrm{W}])$ and later called the generalized Lefschetz number by some authors (e.g. $[\mathrm{FH}])$. The difference is algebraic. We use ordinary conjugacy classes in $\pi_{1}\left(T_{f}\right)$ instead of the so called Reidemeister conjugacy classes in $\pi_{1}(X)$.

2.3. The trace formula and the Lefschetz zeta function. So far $L_{\Gamma}\left(f^{n}\right)$ is defined as a formal sum organizing the index and coordinate information of the periodic orbit classes. Its importance lies in its computability.

Pick a base point $v \in X$ and a path $w$ from $v$ to $f(v)$. Let $G:=\pi_{1}(X, v)$ and let $f_{G}: G \rightarrow G$ be the composition

$$
\pi_{1}(X, v) \stackrel{f_{*}}{\rightarrow} \pi_{1}(X, f(v)) \stackrel{w_{*}}{\rightarrow} \pi_{1}(X, v) .
$$

Let $p: \tilde{X}, \tilde{v} \rightarrow X, v$ be the universal covering. The deck transformation group is identified with $G$. Let $\tilde{f}: \widetilde{X} \rightarrow \widetilde{X}$ be the lift of $f$ such that the reference path $w$ lifts to a path from $\tilde{v}$ to $\tilde{f}(\tilde{v})$. Then for every $g \in G$ we have $\tilde{f} \circ g=f_{G}(g) \circ \tilde{f}$ (cf. [J1, pp. 24-25]).

Assume that $X$ is a finite cell complex and $f: X \rightarrow X$ is a cellular map. Pick a cellular decomposition $\left\{e_{j}^{d}\right\}$ of $X$, the base point $v$ being a 0 -cell. It lifts to a $G$-invariant cellular structure on the universal covering $\widetilde{X}$. Choose an arbitrary lift $\tilde{e}_{j}^{d}$ for each $e_{j}^{d}$. These lifts constitute a free $\mathbb{Z} G$-basis for the cellular chain complex of $\tilde{X}$. The lift $\tilde{f}$ of $f$ is also a cellular map. In every dimension $d$, the cellular chain map $\tilde{f}$ gives rise to a $\mathbb{Z} G$-matrix $\widetilde{F}_{d}$ with respect to the above basis, i.e. $\widetilde{F}_{d}=\left(a_{i j}\right)$ if $\tilde{f}\left(\tilde{e}_{i}^{d}\right)=\sum_{i} a_{i j} \tilde{e}_{j}^{d}, a_{i j} \in \mathbb{Z} G$.

For the mapping torus, take the base point $v$ of $X$ as the base point of $T_{f}$ (recall that $X$ is regarded as embedded in $\left.T_{f}\right)$. Let $\Gamma=\pi_{1}\left(T_{f}, v\right)$. By the van Kampen Theorem, $\Gamma$ is obtained from $G$ by adding a new generator $z$ represented by the loop $\varphi_{(v, 1)} w^{-1}$, and adding the relations $z^{-1} g z=f_{G}(g)$ for all $g \in G$ :

$$
\left.\Gamma=\langle G, z| g z=z f_{G}(g) \text { for all } g \in G\right\rangle \text {. }
$$

Note that the homomorphism $G \rightarrow \Gamma$ induced by the inclusion $X \subset T_{f}$ is not necessarily injective.

In this notation, we can adapt the Reidemeister trace formula $([\mathrm{R}],[\mathrm{W}])$ to our mapping torus setting and get a simple formula.

Trace Formula for Lefschetz numbers. For the Lefschetz numbers we have

$$
L_{\Gamma}\left(f^{n}\right)=\sum_{d}(-1)^{d}\left[\operatorname{tr}\left(z \widetilde{F}_{d}\right)^{n}\right] \in \mathbb{Z} \Gamma_{c},
$$

where $z \widetilde{F}_{d}$ is regarded as a matrix in $\mathbb{Z} \Gamma$.

Suppose a group representation $\rho: \Gamma \rightarrow \mathrm{GL}_{l}(R)$ is given, where $R$ is a commutative ring with unity. Then $\rho$ extends to a ring representation $\rho: \mathbb{Z} \Gamma \rightarrow \mathcal{M}_{l \times l}(R)$, where $\mathcal{M}_{l \times l}(R)$ is the algebra of $l \times l$ matrices in $R$. 
Define the $\rho$-twisted Lefschetz number

$$
L_{\rho}\left(f^{n}\right):=\operatorname{tr}\left(L_{\Gamma}\left(f^{n}\right)\right)^{\rho}=\sum_{\mathbf{O}^{n}} \operatorname{ind}\left(\mathbf{O}^{n}, f^{n}\right) \cdot \operatorname{tr}\left(\operatorname{cd}_{\Gamma}\left(\mathbf{O}^{n}\right)\right)^{\rho} \in R
$$

for every natural number $n$, the summation being over all $n$-orbit classes $\mathbf{O}^{n}$.

We now define the ( $\rho$-twisted) Lefschetz zeta function of $f$ to be the formal power series

$$
\zeta_{\rho}(f):=\exp \sum_{n} L_{\rho}\left(f^{n}\right) \frac{t^{n}}{n} .
$$

It has constant term 1 , so it is in the multiplicative subgroup $1+t R[[t]]$ of the formal power series ring $R[[t]]$.

Clearly $\zeta_{\rho}(f)$ enjoys the same invariance properties as $L_{\Gamma}\left(f^{n}\right)$. As to its computation, we obtain from the trace formula the following

Determinant Formula For the Lefschetz ZETA FunCtion.$\zeta_{\rho}(f)$ is a rational function in $R$.

$$
\zeta_{\rho}(f)=\prod_{d} \operatorname{det}\left(I-t\left(z \widetilde{F}_{d}\right)^{\rho}\right)^{(-1)^{d+1}} \in R(t),
$$

where $\left(z \widetilde{F}_{d}\right)^{\rho}$ means the block matrix obtained from the matrix $z \widetilde{F}_{d}$ by replacing each entry $($ in $\mathbb{Z} \Gamma$ ) with its $\rho$-image (an $l \times l$ matrix), and I stands for suitable identity matrices.

By the trace and determinant formulas and the homotopy invariance, we have the

TWisted Version OF THE LefSChetz FiXed POINT THEOREM. Let $f: X \rightarrow X$ be a map and $\rho: \pi_{1}\left(T_{f}\right) \rightarrow \mathrm{GL}_{l}(R)$ be a representation. If $f$ is homotopic to a fixed point free map $g$, then $L_{\rho}(f)=0$. If $f$ is homotopic to a periodic point free map $g$, then $\zeta_{\rho}(f)=1$.

EXAmple 1 . When $R=\mathbb{Z}$ and $\rho: \Gamma \rightarrow \mathrm{GL}_{1}(\mathbb{Z})=\mathbb{Z}$ is trivial (sending everything to 1 ), then $L_{\rho}(f) \in \mathbb{Z}$ is the ordinary Lefschetz number $L(f)$, and $\zeta_{\rho}(f)$ is the classical Lefschetz zeta function $\zeta(f):=\exp \sum_{n} L\left(f^{n}\right) t^{n} / n$ introduced by Weil.

ExAmple 2. Suppose $H$ is a commutative group and $\rho: \Gamma \rightarrow H$ is a homomorphism. Take $R=\mathbb{Z} H$. Then $\rho$ extends to $\rho: \mathbb{Z} \Gamma \rightarrow \mathrm{GL}_{1}(\mathbb{Z} H)=\mathbb{Z} H$. Then $L_{\rho}\left(f^{n}\right) \in \mathbb{Z} H$ and $\zeta_{\rho}(f)$ is a rational function in $\mathbb{Z} H$.

In particular, we can take $H=H_{1}\left(T_{f}\right)$ to be the abelianization of $\Gamma$, regarded as a multiplicative group. It is the direct product of $\operatorname{coker}\left(f_{*}: H_{1}(X) \rightarrow H_{1}(X)\right)$ (also regarded multiplicatively) with the infinite cyclic group generated by $z$. Let $\rho: \Gamma \rightarrow H$ be the projection. Then $L_{\rho}\left(f^{n}\right) \in \mathbb{Z} H$ is the central invariant of homological Nielsen theory, in which two $n$-orbits are regarded as equivalent if and only if they represent the same homology class in $T_{f}$. The coordinate of such a homological $n$-orbit class lies in $H$. $L_{\rho}\left(f^{n}\right) \in \mathbb{Z} H$ is the formal sum of such coordinates, with integer coefficients the indices of the classes. $\zeta_{\rho}(f) \in \mathbb{Z} H(t)$ is the generating function of the sequence $\left\{L_{\rho}\left(f^{n}\right)\right\}$.

REMARK. Our Lefschetz zeta function is essentially the same as the twisted Lefschetz function of David Fried [Fr]. He first introduced it using $f$-invariant abelianizations of $\pi_{1}(X)$, and showed that it is a certain Reidemeister torsion of the mapping torus $T_{f}$. Then he adopted the Reidemeister torsion approach with respect to a flat vector bundle (which is equivalent to a matrix representation of the fundamental group). 
2.4. Asymptotic invariants. The growth rate of a sequence $\left\{a_{n}\right\}$ of complex numbers is defined by

$$
\underset{n \rightarrow \infty}{\text { Growth }} a_{n}:=\max \left\{1, \limsup _{n \rightarrow \infty}\left|a_{n}\right|^{1 / n}\right\}
$$

which could be infinity. When Growth $a_{n}>1$, we say that the sequence grows exponentially.

We define the asymptotic Nielsen number of $f$ to be the growth rate of the Nielsen numbers

$$
N^{\infty}(f):=\underset{n \rightarrow \infty}{\operatorname{Growth}} N\left(f^{n}\right)=\underset{n \rightarrow \infty}{\operatorname{Growth}} N_{\Gamma}\left(f^{n}\right),
$$

where the second equality is due to the obvious inequality $N_{\Gamma}\left(f^{n}\right) \leq N\left(f^{n}\right) \leq n \cdot N_{\Gamma}\left(f^{n}\right)$. And we define the asymptotic irreducible Nielsen number of $f$ to be the growth rate of the irreducible Nielsen numbers

$$
N I^{\infty}(f):=\underset{n \rightarrow \infty}{\operatorname{Growth}} N I_{\Gamma}\left(f^{n}\right) .
$$

We also define the asymptotic absolute Lefschetz numbers

$$
\begin{aligned}
L^{\infty}(f) & :=\underset{n \rightarrow \infty}{\operatorname{Growth}}\left\|L_{\Gamma}\left(f^{n}\right)\right\|, \\
L I^{\infty}(f) & :=\underset{n \rightarrow \infty}{\text { Growth }}\left\|L I_{\Gamma}\left(f^{n}\right)\right\| .
\end{aligned}
$$

All these asymptotic numbers are finite and share the invariance properties of $L_{\Gamma}\left(f^{n}\right)$.

The asymptotic invariants measure the growth of the number of periodic orbits. In practice, the estimation of these growth rates is often easier than the estimation for a specific period $n$.

A Method of Estimation. Suppose $R=\mathbb{C}$ and $\rho: \Gamma \rightarrow \mathrm{U}(l)$ is a unitary representation. Let $r$ be the minimum modulus of the zeros and poles of the rational function $\zeta_{\rho}(f)$. Then

$$
L^{\infty}(f) \geq \frac{1}{r}
$$

The asymptotic Nielsen number provides a homotopy lower bound for the topological entropy which measures the dynamical complexity of maps.

Entropy Theorem ([I]). Suppose $X$ is a compact polyhedron and $f: X \rightarrow X$ is a map. Then for any map $g: X \rightarrow X$ homotopic to $f$, the topological entropy $h(g) \geq$ $\log N^{\infty}(f)$.

3. Surface homeomorphisms. Thurston's surface theory lies at the foundation of the study of surface homeomorphisms. In $\S 3.1$ we discuss the asymptotic invariants for self-homeomorphisms of aspherical surfacesResults on minimal representatives in isotopy classes are then given. $§ 3.2$ talks about the Nielsen theory for self-homeomorphisms of punctured surfaces which is very useful in applications.

3.1. Compact aspherical surfaces. Let $X$ be a compact connected aspherical surface and let $f: X \rightarrow X$ be a homeomorphism. The main result of this section is easier when $X$ is the disc, the annulus, the Möbius strip, the torus or the Klein bottle. So we shall assume $\chi(X)<0$. 
Thurston Theorem ([T], see also [FLP $]$ ). Every homeomorphism $f: X \rightarrow X$ is isotopic to a homeomorphism $\varphi$ such that either

(1) $\varphi$ is a periodic map, i.e. $\varphi^{m}=i d$ for some $m$; or

(2) $\varphi$ is a pseudo-Anosov map, i.e. there is a number $\lambda>1$ and a pair of transverse measured foliations $\left(\mathfrak{F}^{s}, \mu^{s}\right)$ and $\left(\mathfrak{F}^{u}, \mu^{u}\right)$ such that $\varphi\left(\mathfrak{F}^{s}, \mu^{s}\right)=\left(\mathfrak{F}^{s}, \frac{1}{\lambda} \mu^{s}\right)$ and $\varphi\left(\mathfrak{F}^{u}, \mu^{u}\right)=\left(\mathfrak{F}^{u}, \lambda \mu^{u}\right) ;$ or

(3) $\varphi$ is a reducible map, i.e. there is a system of disjoint simple closed curves $\gamma=$ $\left\{\gamma_{1}, \cdots, \gamma_{k}\right\}$ in int $X$ such that $\gamma$ is invariant by $\varphi$ (but the $\gamma_{i}$ 's may be permuted) and $\gamma$ has a $\varphi$-invariant tubular neighborhood $U$ such that each component of $X \backslash U$ has negative Euler characteristic and on each (not necessarily connected) $\varphi$-component of $X \backslash U, \varphi$ satisfies (1) or (2).

The $\varphi$ above is called the Thurston canonical form of $f$. In (3) it can be chosen so that some iterate $\varphi^{m}$ is a generalized Dehn twist on each component of $U$. Such a $\varphi$, as well as the $\varphi$ in (1) or (2), is called standard in [JG, §3.1]. Its fixed point classes are well understood. E.g. "almost every" essential fixed point class has index \pm 1 , so that we have the inequality

$$
|L(f)-\chi(M)| \leq N(f)-\chi(M) .
$$

Since iterates of standard maps are standard, we can also obtain information about the periodic orbit classes. E.g. when $n>-2 \chi(X)$, every essential irreducible $n$-point class has index \pm 1 , hence the equality

$$
\left\|L I_{\Gamma}\left(f^{n}\right)\right\|=n \cdot N I_{\Gamma}\left(f^{n}\right) .
$$

We have the following results.

ASYMPTOTIC INVARIANTS.

$$
N I^{\infty}(f)=N^{\infty}(f)=L I^{\infty}(f)=L^{\infty}(f)=\lambda,
$$

where $\lambda$ is the largest stretching factor of the pseudo-Anosov pieces in the Thurston canonical form of $f(\lambda:=1$ if there is no pseudo-Anosov piece).

For the question of isotopy minimum, by a careful construction we have

Minimum fixed points ([JG]). Every homeomorphism $f: X \rightarrow X$ of a closed surface is isotopic to a homeomorphism $\varphi: X \rightarrow X$ which has the minimum number of fixed points in the isotopy class. This number is $N(f)$ if $X$ is orientable and $f$ is orientation preserving. In the general case the minimum number is the relative Nielsen number $N(f ; X, \partial X)$ introduced by Schirmer $[\mathrm{S}]$.

Minimum PeRIOdic orbits, ORIEnted CASE (cf. [Bo2]). Suppose $X$ is orientable. Every orientation preserving homeomorphism $f: X \rightarrow X$ is isotopic to a homeomorphism $\varphi: X \rightarrow X$ which has, for every period $n$, the minimum number of primary $n$-orbits in the isotopy class. This minimum number is $N I_{\Gamma}\left(f^{n}\right)$ for every $n$.

For a general surface homeomorphism, we can prove a weaker result by combining the techniques of [JG] and [Bo2]. 
Minimum Periodic orbits, General CaSe. Suppose $f: X \rightarrow X$ is a surface homeomorphism. Then

(1) $f$ is isotopic to a homeomorphism $\varphi: X \rightarrow X$ which has the minimum number of primary $n$-orbits in the isotopy class, for all $n>-3 \chi(X)$.

(2) for any given period $n, f$ is isotopic to a homeomorphism $\varphi_{n}: X \rightarrow X$ whose number of primary n-orbits is the minimum in the isotopy class.

The minimum number referred to is $N I_{\Gamma}\left(f^{n}\right)$ for all $n$.

The Thurston theory is very useful in applications because it is computable. Algorithms for determining the Thurston canonical form of any given surface homeomorphism have been developed. We don't have time to describe them here. The interested reader is referred to the original papers [BH1, BH2, BGN, FM, Lo].

REMARK. When $f: X \rightarrow X$ is a homeomorphism of a surface $X$, the mapping torus $T_{f}$ is a 3-manifold. The following geometric notions were introduced in dynamics [AF].

Two primary $n$-orbits of $f$ are strong Nielsen equivalent if and only if their time- $n$ orbit curves are freely isotopic (instead of homotopic) as closed curves in $T_{f}$. The equivalent classes are called strong $n$-orbit classes.

An isotopy $\left\{h_{t}\right\}: f \simeq g: X \rightarrow X$ between two homeomorphisms naturally induces a homeomorphism $H: T_{f} \rightarrow T_{g}$ between the mapping tori. A strong $n$-orbit class $A$ of $f$ corresponds to a strong $n$-orbit class $B$ of $g$ under $\left\{h_{t}\right\}$, if the isotopy class of the former orbit curves corresponds to that of the latter orbit curves under $H$. In terms of paths, this means there is an isotopy $\left\{h_{t}^{\prime}\right\}: f \simeq g: X \rightarrow X$ (which is required to be a deformation of the given isotopy $\left\{h_{t}\right\}$ ), and a path $c: I \rightarrow X$ from a point $a \in A$ to a point $b \in B$ such that, for all $t \in I, c(t)$ is in a primary $n$-orbit of $h_{t}^{\prime}$. A strong $n$-orbit class of $f$ is unremovable if it corresponds to some strong $n$-orbit class under any isotopy of $f$.

These notions were intended as a refinement to the Nielsen theory of periodic orbits in that isotopy is much stronger than homotopy for closed curves in 3-manifolds, hence an orbit class splits into a disjoint union of strong orbit classes. But the minimality results stated above indicate that an unremovable strong $n$-orbit class coincides with an essential irreducible $n$-orbit class. Thus, as far as the isotopy minimality problem is concerned, this refinement is not necessary.

3.2. Punctured surfaces. Let $X$ be a connected compact surface and let $P$ be a nonempty finite set of points (punctures) in the interior of $X$. Assume that $\chi(X)-|P|<0$ where $|P|$ denotes the cardinality of $P$. Let $f: X, P \rightarrow X, P$ be a homeomorphism. We shall be concerned with periodic orbits of $f$ in $X \backslash P$. We shall refer to the punctured $\operatorname{map} f \backslash P: X \backslash P \rightarrow X \backslash P$.

Thurston's theory works for punctured surfaces [FLP]. Although the space $X \backslash P$ is non-compact, Nielsen fixed point theory (which is for compact polyhedra) can be adapted to work in this setting of punctured homeomorphisms. For details see [J3]. Thus everything in $\S 3.1$ has a punctured version and the same statements hold.

As a sample, let $M I^{n}(f \backslash P)$ be the minimum number of primary $n$-orbits of $h \backslash P$ for any homeomorphism $h: X, P \rightarrow X, P$ isotopic to $f$ rel $P$, and define $M I^{\infty}(f \backslash P)$ to be the growth rate $\operatorname{Growth}_{n \rightarrow \infty} M I^{n}(f \backslash P)$. Then we have: 
PunCTURED ASYMPtotic InVARIANTS.

$$
M I^{\infty}(f \backslash P)=N^{\infty}(f \backslash P)=\lambda,
$$

where $\lambda$ is the largest stretching factor of the pseudo-Anosov pieces in the Thurston canonical form of the punctured map $f \backslash P(\lambda:=1$ if there is no pseudo-Anosov piece).

Consequently, as in $\S 2.4, N^{\infty}(f \backslash P)$ can also be estimated if we are given a unitary representation of $\Gamma=\pi_{1}\left(T_{f \backslash P}\right)=\pi_{1}\left(T_{f} \backslash T_{f \mid P}\right)$.

Entropy Theorem. For any homeomorphism $f: X, P \rightarrow X, P$, we have

$$
h(f) \geq \log N^{\infty}(f \backslash P) .
$$

Equality holds when $f \backslash P$ is in Thurston canonical form.

The theory above has many concrete applications in dynamics. See [HJ] and [J3] for examples. The next section will discuss the punctured disk in more detail.

4. The punctured disk. As a model of the type of problems that can be tackled by Nielsen theory, we focus on the study of orientation preserving self-homeomorphisms of the 2-dimensional disk. Braid groups and their representations play a central role in the calculation of the Nielsen theory invariants.

4.1. The setting. Let $X$ be the disk $D^{2}$. Let $f: D^{2} \rightarrow D^{2}$ be an orientation preserving homeomorphism and let $P \subset \operatorname{int} D^{2}$ be a set of $r$ points with $f(P)=P$. Let $H=\left\{h_{t}\right\}_{t \in I}$ : $i d \simeq f: D^{2} \rightarrow D^{2}$ be an isotopy from the identity map to $f$.

The set $\mathcal{S}:=\left\{\left(h_{t}(x), t\right) \in X \times I \mid x \in P\right\}$ is a geometric braid in $D^{2} \times I$ which represents a braid $\sigma$ in Artin's $r$-string braid group $B_{r}$ (cf. [Bi, p. 6] or [M, Ch. 4]).

Since we shall allow isotopy rel $P$, without loss we may assume that $f$ and $H$ are the identity on the boundary $\partial D^{2}$. The isotopy $H$ is not uniquely determined by the map $f$, but up to an isotopy from the identity map to itself, thus the braid $\sigma$ is determined up to multiplication by "full twists". Note that the center $Z$ of $B_{r}$ is the infinite cyclic subgroup generated by the "full twist" produced by the $2 \pi$-rotation of the plane (cf. [Bi, p. 28]). So the braid $\sigma$ is uniquely determined $\bmod Z$.

Let $M$ be the punctured disk $D^{2} \backslash P$. Then $f \backslash P$ is a homeomorphism $M \rightarrow M$. The automorphism $f_{G}: \pi_{1}(M) \rightarrow \pi_{1}(M)$ can be computed in terms of the braid data $\sigma$. Then representations of $\Gamma=\pi_{1}\left(T_{f} \backslash T_{f \mid P}\right)$ can be found and Lefschetz zeta functions computed, so that the estimation methods in $\S 2.4$ can be applied to obtain information on periodic orbits of $f \backslash P$.

4.2. The algebraic recipe for computations. The fundamental group $G=\pi_{1}(M)$ is the free group $F_{r}$ of rank $r$, with standard generators $\left\{a_{1}, \cdots, a_{r}\right\}$. Artin's braid group $B_{r}$ has standard generators $\left\{\sigma_{1}, \cdots, \sigma_{r-1}\right\}$ and relations $\sigma_{i} \sigma_{i+1} \sigma_{i}=\sigma_{i+1} \sigma_{i} \sigma_{i+1}$ for $1 \leq i<r-1$ and $\sigma_{i} \sigma_{j}=\sigma_{j} \sigma_{i}$ if $|i-j|>1$.

The braid $\sigma \in B_{r}$ determines the isotopy class of the map $f$ by "sliding the plane down the braid", hence it determines the automorphism $f_{\sigma}: G \rightarrow G$. The correspondence from $\sigma$ to $f_{\sigma}$ is actually a faithful representation of $B_{r}$ into the (right) automorphism 
group Aut $F_{r}$, given by (cf. [Bi, p. 25] or [M, p. 86])

$$
\sigma_{i}:\left\{\begin{array}{l}
a_{i} \quad \mapsto a_{i} a_{i+1} a_{i}^{-1} \\
a_{i+1} \mapsto a_{i}, \\
a_{j} \mapsto a_{j} \quad \text { if } j \neq i, i+1 .
\end{array}\right.
$$

The automorphism $f_{\sigma}: G \rightarrow G$ induced by $f$ is determined by the images $a_{i}^{\prime}:=f_{\sigma}\left(a_{i}\right)$, $i=1, \cdots, r$. The fundamental group $\Gamma=\pi_{1}\left(T_{f \mid M}\right)$ has a presentation

$$
\Gamma=\left\langle a_{1}, \cdots, a_{r}, z \mid a_{i} z=z a_{i}^{\prime}, i=1, \cdots, r\right\rangle .
$$

There is a natural abelian representation of $\Gamma$. The conjugacy classes of the generators $\left\{a_{i}\right\}$ are represented by small circles around the punctures, hence permuted by the action of the braids. So we can always define a homomorphism $\theta: \Gamma \rightarrow A$, where $A$ is the infinite cyclic group $A:=\langle a\rangle$, by sending all $a_{i}$ to the generator $a$ and sending $z$ to 1 . The group algebra $\mathbb{Z} A$ is the ring $\mathbb{Z}\left[a^{ \pm 1}\right]$ of integral Laurent polynomials in the variable $a$. The homomorphism $\theta: \Gamma \rightarrow A$ can be regarded as a representation $\theta: \Gamma \rightarrow \mathrm{GL}\left(1, \mathbb{Z}\left[a^{ \pm 1}\right]\right)$.

Now $M$ has the homotopy type of a bouquet $X^{\prime}$ of $r$ circles corresponding to the basis elements, and $f$ has the homotopy type of a map $f^{\prime}: X^{\prime} \rightarrow X^{\prime}$ which induces the same homomorphism $G \rightarrow G$. By the homotopy type invariance of the invariants, we can replace $f$ with $f^{\prime}$ in computations.

As pointed out in $[\mathrm{FH}]$, in the trace formula the matrices of the lifted chain map $\tilde{f}^{\prime}$ are

$$
\tilde{F}_{0}=(1), \quad \tilde{F}_{1}=D:=\left(\frac{\partial a_{i}^{\prime}}{\partial a_{j}}\right)
$$

where $D$ is the Jacobian matrix in Fox calculus (see [Bi, §3.1] or [M, Ch. 8] for an introduction). Then, by the trace formula, in $\mathbb{Z} \Gamma_{c}$ we have

$$
L_{\Gamma}(f)=[z]-\sum_{i=1}^{r}\left[z \frac{\partial a_{i}^{\prime}}{\partial a_{i}}\right], \quad L_{\Gamma}\left(f^{n}\right)=\left[z^{n}\right]-\left[\operatorname{tr}(z D)^{n}\right] .
$$

Under the representation $\theta: \Gamma \rightarrow A$, we have

$$
\begin{gathered}
L_{\theta}(f)=\operatorname{tr} z^{\theta}-\operatorname{tr}(z D)^{\theta} \in \mathbb{Z}\left[a^{ \pm 1}\right], \\
\zeta_{\theta}(f)=\frac{\operatorname{det}\left(I-t(z D)^{\theta}\right)}{\operatorname{det}\left(I-t z^{\theta}\right)} \in \mathbb{Z}\left[a^{ \pm 1}\right](t) .
\end{gathered}
$$

Thus, the Fox Jacobian $D$ of $f_{\sigma}$ is the key to the Lefschetz zeta function. The correspondence from $\sigma$ to $(z D)^{\theta}$ is exactly the famous Burau representation of the braid group into matrices in $\mathbb{Z}\left[a^{ \pm 1}\right]$ : (cf. [Bi, p. 118] or [M, p. 193])

$$
B: B_{r} \rightarrow \mathrm{GL}\left(r, \mathbb{Z}\left[a^{ \pm 1}\right]\right), \quad \sigma_{i} \mapsto\left(\begin{array}{cccc}
I & & & \\
& 1-a & a & \\
& 1 & 0 & \\
& & & I
\end{array}\right) \leftarrow i \text {-th row }
$$

which is reducible to the reduced Burau representation ([Bi, p. 121] or [M, p. 225]) 


$$
B^{\prime}: B_{r} \rightarrow \mathrm{GL}\left(r-1, \mathbb{Z}\left[a^{ \pm 1}\right]\right), \quad \sigma_{i} \mapsto\left(\begin{array}{ccccc}
I & & & & \\
& 1 & 0 & 0 & \\
& a & -a & 1 & \\
0 & 0 & 1 & \\
& & & & I
\end{array}\right) \leftarrow i \text {-th row. }
$$

Hence from $\S 2.3$ we see

$$
\begin{gathered}
L_{\theta}\left(f^{n}\right)=1-\operatorname{tr} B(\sigma)^{n}=-\operatorname{tr} B^{\prime}(\sigma)^{n} \in \mathbb{Z}\left[a^{ \pm 1}\right], \\
\zeta_{\theta}(f)=\frac{\operatorname{det}(I-t B(\sigma))}{1-t}=\operatorname{det}\left(I-t B^{\prime}(\sigma)\right) \in \mathbb{Z}\left[a^{ \pm 1}\right](t) .
\end{gathered}
$$

4.3. Exponential growth and topological entropy. By $\S 2.4$ we know the number of periodic orbits grows exponentially and the topological entropy is positive if we have $N^{\infty}(f \backslash P)>1$.

In $\S 4.2$, take $a$ to be a complex number of modulus 1 . We obtain a unitary representation $\rho: \Gamma \rightarrow \mathrm{U}(1)$. Now $(z D)^{\rho}$ is obtained from the matrix $B(\sigma)$ in $\mathbb{Z}\left[a^{ \pm 1}\right]$ by regarding it as a function of the unimodular complex variable $a$. Then we have the twisted invariants

$$
L_{\rho}\left(f^{n}\right)=-\operatorname{tr} B^{\prime}(\sigma)^{n} \in \mathbb{C}, \quad \zeta_{\rho}(f)=\operatorname{det}\left(I-t B^{\prime}(\sigma)\right) \in \mathbb{C}(t) .
$$

Hence from the lower estimation of $\S 2.4$ we get

$$
N^{\infty}(f \backslash P) \geq \max _{|a|=1}\left\{\text { spectral radius of } B^{\prime}(\sigma)\right\} .
$$

The case $r=3$ attracts most attention. It can be shown [Ko1] that

$$
N^{\infty}(f \backslash P)=\text { spectral redius of }\left.B^{\prime}(\sigma)\right|_{a=-1} .
$$

In geometric terms, we have

Generic Exponential GRowth For 3-Braids (Cf. [Ma], [J2]). The number of $n$-orbit classes of $f \backslash P$ grows exponentially in $n$ (i.e. $N^{\infty}(f \backslash P)>1$ ), with the only exception when the 3-braid $\sigma$ is conjugate in $B_{3} / Z$ to $\sigma_{1}^{m}(m \in \mathbb{Z}),\left(\sigma_{1} \sigma_{2}\right)^{ \pm 1}$ or $\sigma_{1} \sigma_{2} \sigma_{1}$.

Here $Z$ is the center of $B_{3}$, the infinite cyclic group generated by the full twist $\left(\sigma_{1} \sigma_{2}\right)^{3}=\left(\sigma_{1} \sigma_{2} \sigma_{1}\right)^{2}$. Note that the only exceptional 3-braids are the simplest ones that would be called twists rather than braids in non-mathematical language. Thus the dynamical phenomenon "period three implies chaos" in dimension 1, although no longer true in dimension 2 , still persists in a subtle way.

As a concrete example, look at the 3-braid $\sigma=\sigma_{1} \sigma_{2}^{-1} \in B_{3}$ discussed in [GST]. One can calculate that

$$
\zeta_{\theta}(f)=\operatorname{det}\left(I-t B^{\prime}(\sigma)\right)=1-\left(1-a-a^{-1}\right) t+t^{2} .
$$

Take $a=-1$, then we get the zeta function $\zeta_{\rho}(f)=1-3 t+t^{2}$ and its smallest root is $r=(3-\sqrt{5}) / 2$. Hence we get

$$
M I^{\infty}(f \backslash P)=N^{\infty}(f \backslash P)=(3+\sqrt{5}) / 2, \quad h(f) \geq \log ((3+\sqrt{5}) / 2) .
$$

4.4. Linking of periodic orbits. Now consider the setting of $\S 4.1$ from a geometric point of view. Under the isotopy $H=\left\{h_{t}\right\}_{t \in I}: i d \simeq f: D^{2} \rightarrow D^{2}$, the punctures $P$ sweep out the geometric braid $\mathcal{S}:=\left\{\left(h_{t}(x), t\right) \in D^{2} \times I \mid x \in P\right\}$ in the cylinder $D^{2} \times I$ which represents the $r$-braid $\sigma \in B_{r}$. Identify the top and bottom of the cylinder via the 
identity map of $D^{2}$ to form the solid torus $T=D^{2} \times S^{1}$, and embed it in the Euclidean space $\mathbb{R}^{3}$ in an unknotted way. (Note that such embeddings are not unique up to isotopy but may differ by framing.) Then $\mathcal{S}$ becomes an oriented link $\mathcal{P}$ (the "closed braid" of the braid $\sigma$ ) lying in $T$. Likewise, an $n$-point $x$ of $f$ gives rise to an oriented closed curve $\mathcal{O}_{x}$ (primary if $x$ is a primary $n$-point) wrapping $n$ times around $T$. So we can study the geometry of the link in $T$ consisting of $\mathcal{P}$ and $\mathcal{O}_{x}$. We shall say that $\mathcal{O}_{x}$ is linked to $\mathcal{P}$ if $\mathcal{O}_{x}$ in $T \backslash \mathcal{P}$ is not homotopic to a closed curve in the boundary $\partial T$.

REMARK. The isotopy $H$ gives rise to a homeomorphism from the mapping torus $T_{f}$ to the solid torus $T$, which sends the link $T_{f \mid P} \subset T_{f}$ to the link $\mathcal{P} \subset T$ and sends the closed curve $\varphi_{(x, n)}$ to the closed curve $\mathcal{O}_{x}$. So we may alternatively think in terms of the mapping torus.

Of particular interest is the case when $P$ is a single periodic orbit $(r \geq 2$ and $f$ acts transitively on $P$ ) and $x$ is a fixed point $(n=1)$. Is there always a fixed point $x$ whose orbit $\mathcal{O}_{x}$ is linked to $\mathcal{P}$ ? The answer is yes, as first shown in [Ko2]. The proof is actually very simple. Collapsing $\partial D^{2}$ to a point $\infty$ and blowing up the punctures $P$, we form a sphere with $r$ holes and extend $f$ to $g$. Since $g$ permutes the boundary curves of the holes, the Lefschetz number of $g$ is easily seen to be 2 . From $\S 3.1$ we see $N(g) \geq L(g)=2$. Thus $g$ has at least one fixed point $x$ that is in a different fixed point class than $\infty$. This $x$ must be a fixed point of $f$ and has the required property.

We can also consider the linking number between $\mathcal{O}_{x}$ and $\mathcal{P}$. In view of the framing problem mentioned above, strictly speaking this linking number is well defined only $\bmod n r$.

The linking number is related to the Burau representation and the Lefschetz number $L_{\theta}\left(f^{n}\right)=-\operatorname{tr} B^{\prime}(\sigma)^{n} \in \mathbb{Z}\left[a^{ \pm 1}\right]$ discussed in $\S 4.2$. Reviewing all the relevant definitions, we can see that a nonzero term $k a^{\ell}$ in $L_{\theta}\left(f^{n}\right)$ guarantees the existence of an essential $n$-orbit $\mathcal{O}_{x}$ whose linking number with $\mathcal{P}$ is exactly the exponent $\ell$. (And the coefficient $k$ is the total contribution from the indices of all such $n$-orbits.) This connection was first noticed in [Ma].

Franks has posed the following question which is known as the linking number problem (see [BF, p. 24]): Suppose $P$ is a single periodic orbit of $f$. Does there exist a fixed point of $f$ about which the orbit $P$ has nonzero linking number?

This problem fits into our setting above with $r \geq 2, n=1$ and $f$ acts transitively on $P$. If $L_{\theta}(f)=-\operatorname{tr} B^{\prime}(\sigma) \in \mathbb{Z}\left[a^{ \pm 1}\right]$ has a term with nonzero exponent, then the answer to Franks' question is yes. In this way it is proved in $[\mathrm{Gu}]$ that there exists a periodic orbit $Q$ of $f$ of period $n<\left\lceil\frac{r}{2}\right\rceil$ such that the linking number of $P$ about $Q$ is nonzero. So the answer to the linking number problem is known to be yes for $r \leq 4$. For larger $r$ it is still open.

4.5. Braiding of periodic orbits. We have seen that in our setting of $\S 4.1$, periodic orbits appear as braids. Strictly speaking, the braid is determined only up to multiplication by full twists and up to conjugacy in the appropriate braid group. The notion of braid type is introduced to reflect this geometric indeterminacy. The set of all braid types will be denoted by BT. 
By analogy with the Sharkovskiu ordering in 1-dimensional dynamics, a partial order was introduced into BT by Boyland $[\mathrm{BF}]$. Let $\beta, \beta^{\prime}$ be braid types. We say $\beta$ forces $\beta^{\prime}$, write $\beta \succcurlyeq \beta^{\prime}$, if any orientation preserving homeomorphism of the disk that has a set of periodic orbits representing $\beta$ must have a set of periodic orbits representing $\beta^{\prime}$. This is clearly a partial order.

The algorithmic approach to surface homeomorphisms (mentioned in §3.1) is clearly very useful in the study of braid types and the forcing order. We restrict ourselves to comments from an algebraic point of view.

The abelianized Lefschetz zeta function is equivalent to the Alexander polynomial of the link obtained by closing the braid, so it is too weak for determining the braid type. The Lefschetz zeta function associated to a representation corresponds to the twisted Alexander polynomial (see [Li, JW, Wd]) whose strength in knot theory is being unveiled (see $[\mathrm{KL}])$. The Lefschetz numbers $L_{\Gamma}\left(f^{n}\right) \in \mathbb{Z} \Gamma_{c}$, without the loss caused by representations, should contain more information about the braid types, for which new algebraic tools are needed.

5. The set of periods. Let $f: X \rightarrow X$ be a map. We denote by $\operatorname{Per} f$ the set of periods of all primary periodic orbits of $f$. The topology of the space $X$ and the homological and homotopical properties of the map $f$ will impose restrictions to the set Per $f$.

There are many interesting questions about the set Per $f$.

5.1. Degree of fixed point freedom. Following Nielsen [N3], we define the degree of fixed point freedom of a map $f$, denoted $D F(f)$, to be the maximum integer $m$ such that $f$, $f^{2}, \ldots f^{m-1}$ are all fixed point free. In other words,

$$
D F(f):=\min \operatorname{Per} f .
$$

It is understood that $D F(f)=\infty$ if Per $f$ is empty, i.e. if $f$ has no periodic orbits.

We then define the degree of freedom for homeomorphisms of a space $X$, denoted $D F H(X)$, to be the maximum of $D F(f)$ for all self-homeomorphisms $f: X \rightarrow X$ :

$$
D F H(X)=\max \{D F(f) \mid f \text { a self-homeomorphism of } X\} .
$$

When $X$ is an orientable manifold, similarly define $D F H_{+}(X)$ and $D F H_{-}(X)$ to be the maximum of $D F(f)$ for all orientation preserving and orientation reversing selfhomeomorphisms $f: X \rightarrow X$ respectively.

Fuller, in [Fu1], proved the following result; see also [Ha] and [B, p. 45].

FUlLer THEOREM. Let $f$ be a homeomorphism of a compact polyhedron $X$ onto itself. If the Euler characteristic $\chi(X) \neq 0$, then $f$ has a periodic point with period not greater than the maximum of $\beta_{\text {odd }}:=\sum_{k \text { odd }} \beta_{k}(X)$ and $\beta_{\text {even }}:=\sum_{k \text { even }} \beta_{k}(X)$, where $\beta_{k}(X)$ denotes the $k$-th Betti number of $X$.

Hence, for compact connected manifolds,

$$
\operatorname{DFH}(X)= \begin{cases}\max \left\{\beta_{\text {odd }}, \beta_{\text {even }}\right\} & \text { if } \chi(X) \neq 0, \\ \infty & \text { if } \chi(X)=0 .\end{cases}
$$


For closed surfaces, there is an earlier result by Nielsen [N3] which was recently extended by Dicks-Llibre [DL] and Wang [Wa2]. We shall denote the orientable closed surface of genus $g$ by $F_{g}$, and denote the nonorientable closed surface of genus $q$ by $N_{q}$.

Degree of Freedom, Closed surfaces.

(1) $\mathrm{DFH}_{+}\left(F_{g}\right)=2 g-2$ if $g \geq 2$.

(2) $\mathrm{DFH}_{-}\left(F_{g}\right)= \begin{cases}2 g-2 & \text { if } g>2, \\ 4 & \text { if } g=2 \text {. }\end{cases}$

(3) $\operatorname{DFH}\left(N_{q}\right)= \begin{cases}q-2 & \text { if } q>3 \\ 2 & \text { if } q=3\end{cases}$

The closed surfaces left out are the simplest ones with $\chi \geq 0$ for which the answer is well known.

Similar questions for surfaces with boundary are being studied. A new result of Moira Chas $[\mathrm{C}]$ gives upper bounds that are independent of the number of boundary components.

Degree of Freedom, BOUnded SuRfaces. Let $F_{g, b}$ be the orientable surface of genus $g$ with $b$ boundary components, $g \geq 2$ and $b>0$. Then

(1) $\mathrm{DFH}_{+}\left(F_{g, b}\right) \leq 4 g+2$; equality holds when $b \geq 6 g+6$.

(2) $D F H_{-}\left(F_{g, b}\right) \leq \begin{cases}4 g-4 & \text { if } g \text { is odd, equality holds when } b \geq 6 g-6 \\ 4 g+4 & \text { if } g \text { is even, equality holds when } b \geq 6 g+10 \text {. }\end{cases}$

Note that the upper bounds given are exactly the maximum order of periodic maps on the closed surfaces (cf. [Wa1]).

5.2. Minimal set of periods. Define the minimal set of periods in the homotopy class of the map $f: X \rightarrow X$ by

$$
\text { MPer } f:=\bigcap_{g \simeq f} \operatorname{Per} g .
$$

The case of surface homeomorphisms is studied in [FL]. The complete answer has been worked out for the 2-torus $T^{2}$ in [ABLSS], and for the 3 -torus $T^{3}$ in [JL]. For higher dimensional tori, we have the following general information.

Characterization of MPer $f$. Let $f: T^{r} \rightarrow T^{r}$ be a torus map. Then the following three conditions are equivalent:

(1) $m \notin \operatorname{MPer} f$;

(2) $N I_{\Gamma}\left(f^{m}\right)=0$;

(3) either $N\left(f^{m}\right)=0$ or $N\left(f^{m}\right)=N\left(f^{m / p}\right)$ for some prime factor $p$ of $m$.

The condition (2) was proved in [Y], the sharper condition (3) was given in [JL].

Trichotomy. Let $f: T^{r} \rightarrow T^{r}$ be a torus map. Then MPer $f$ is in one of the following three (mutually exclusive) types, where the letters $E, F$ and $G$ are chosen to represent "empty", "finite" and "generic" respectively:

(E) MPer $f$ is empty if and only if $\operatorname{det}(I-A)=0$; 
(F) MPer $f$ is nonempty but finite if and only if all the eigenvalues of $A$ are either zero or roots of unity;

(G) MPer $f \subset T_{A}$ is infinite and $T_{A} \backslash$ MPer $f$ is finite.

Here $A$ is the matrix of the induced homology homomorphism $f_{*}: H_{1}\left(T^{r}\right) \rightarrow H_{1}\left(T^{r}\right)$, and $T_{A}=\left\{n \in \mathbf{N} \mid \operatorname{det}\left(I-A^{n}\right) \neq 0\right\}$.

Moreover, there are finite sets $P(r), Q(r)$ of integers, depending only on the dimension $r$, such that MPer $f \subset P(r)$ in Type $F$ and $T_{A} \backslash$ MPer $f \subset Q(r)$ in Type $G$.

The trichotomy was discussed in [ABLSS], the existence of uniform bounds $P(r)$ and $Q(r)$ for each dimension $r$ was shown in [JL].

One would like to see similar results for other spaces.

\section{References}

[ABLSS] L. Alsedì, S. Baldwin, J. Llibre, R. Swanson and W. Szlenk, Torus maps and Nielsen numbers, in: Nielsen Theory and Dynamical Systems, Ch. McCord (ed.), Contemp. Math. 152, Amer. Math. Soc., Providence, 1993, 1-7; Minimal sets of periods for torus maps via Nielsen numbers, Pacific J. Math. 169 (1995), 1-32.

[AF] D. Asimov and J. Franks, Unremovable closed orbits, in: Geometric Dynamics, J. Palis Jr. (ed.), Lecture Notes in Math. 1007, Springer, Berlin, 1983, 22-29.

[BGN] D. Benardete, M. Gutierrez and Z. Nitecki, A combinatorial approach to reducibility of mapping classes, in: Mapping Class Groups and Moduli Spaces of Riemann Surfaces, C.-F. Bödigheimer, R. M. Hain (eds.), Contemp. Math. 150, Amer. Math. Soc., Providence, 1993, 1-31; Braids and the Nielsen-Thurston classification, J. Knot Theory Ramif. 4 (1995), 549-618.

[BH1] M. Bestvina and M. HAndel, Train tracks and automorphisms of free groups, Ann. of Math. 135 (1992), 1-51.

[BH2] M. Bestvina and M. HANDEL, Train tracks for surface automorphisms, Topology 34 (1995), 109-140.

[Bi] J. S. Birman, Braids, Links, and Mapping Class Groups, Ann. Math. Stud. 82, Princeton Univ. Press, Princeton, 1974.

[Bo1] P. Boyland, Topological methods in surface dynamics, Topology Appl. 58 (1994), 223-298.

[Bo2] P. Boyland, Isotopy stability of dynamics on surfaces, preprint, in: Geometry and Topology in Dynamics, M. Barge and K. Kuperberg (eds.), Contemp. Math. 246, Amer. Math. Soc., 1999, 17-46.

[BF] P. Boyland and J. Franks, Notes on Dynamics of Surface Homeomorphisms, informal lecture notes, Univ. of Warwick, Coventry, 1989.

[Br] L. Brouwer, Über die Minimalzahl der Fixpunkte bei den Klassen von eindeutigen stetigen Transformationen der Ringflächen, Math. Ann. 82 (1921), 94-96.

[B] R. F. Brown, The Lefschetz Fixed Point Theorem, Scott-Foresman, Chicago, 1971.

[C] M. CHAS, Minimum periods of homeomorphisms on orientable surfaces, thesis, UAB Barcelona, 1998.

[DL] W. DiCKs and J. LLIBRE, Orientation-preserving self-homeomorphisms of the surface of genus two have points of period at most two, Proc. Amer. Math. Soc. 124 (1996), 1583-1591. 
[D] A. Dold, Lectures on Algebraic Topology, Springer, Berlin, 1972, 1980.

[FH] E. FAdell and S. Husseini, The Nielsen number on surfaces, in: Topological Methods in Nonlinear Functional Analysis, S. P. Singh et al. (eds.), Contemp. Math. 21, Amer. Math. Soc., Providence, 1983, 59-98.

[FlP] A. Fathi, F. Laudenbach and V. Poenaru, Travaux de Thurston sur les surfaces, Astérisque 66-67 (1979).

[FL] J. Franks and J. Llibre, Periods of surface homeomorphisms, in: Continuum Theory and Dynamical Systems, M. Brown (ed.), Contemp. Math. 117, Amer. Math. Soc., Providence, 1991, 63-77.

[FM] J. Franks and M. Misiurewicz, Cycles for disk homeomorphisms and thick trees, in: Nielsen Theory and Dynamical Systems, Ch. McCord (ed.), Contemp. Math. 152, Amer. Math. Soc., Providence, 1993, 69-139.

[Fr] D. Fried, Periodic points and twisted coefficients, in: Geometric Dynamics, J. Palis Jr. (ed.), Lecture Notes in Math. 1007, Springer, Berlin, 1983, 261-293; Homological identities for closed orbits, Invent. Math. 71 (1983), 419-442; Entropy and twisted cohomology, Topology 25 (1986), 455-470; Lefschetz formulas for flows, in: The Lefschetz Centennial Conference, Part III: Proceedings on Differential Equations, A. Verjovsky (ed.), Contemp. Math. 58.III, Amer. Math. Soc., Providence, 1987, 19-69.

[Fu1] F. B. Fuller, The existence of periodic points, Ann. of Math. 57 (1953), 229-230.

[Fu2] F. B. FulleR, The treatment of periodic orbits by the methods of fixed point theory, Bull. Amer. Math. Soc. 72 (1966), 838-840; An index of fixed point type for periodic orbits, Amer. J. Math. 89 (1967), 133-148.

[GST] J.-M. Gambaudo, S. van Strien and C. Tresser, Vers un ordre de Sarkovskii pour les plongements du disque préservant l'orientation, C. R. Acad. Sci. Paris Sér. I 310 (1990), 291-294.

[GN] R. GEOGHEGAN and A. NiCAS, Lefschetz trace formulae, zeta functions and torsion in dynamics, in: Nielsen Theory and Dynamical Systems, Ch. McCord (ed.), Contemp. Math. 152, Amer. Math. Soc., Providence, 1993, 141-157; Trace and torsion in the theory of flows, Topology 33 (1994), 683-719.

[Gu] J. GuAschI, Representations of Artin's braid groups and linking numbers of periodic orbits, J. Knot Theory Ramif. 4 (1995), 197-212.

[Ha] B. HALpern, Fixed points for iterates, Pacific J. Math. 25 (1968), 255-275.

[H] H. Hopf, A new proof of the Lefschetz formula on invariant points, Proc. Nat. Acad. Sci. USA 14 (1928), 149-153; Über die algebraische Anzahl von Fixpunkten, Math. Z. 29 (1929), 493-524.

[HJ] H.-H. HuAng and B.-J. JiAng, Braids and periodic solutions, in: Topological Fixed Point Theory and Applications (Tianjin, 1988), B. Jiang (ed.), Lecture Notes in Math. 1411, Springer, Berlin, 1989, 107-123.

[I] N. V. Ivanov, Entropy and the Nielsen numbers, Soviet Math. Dokl. 26 (1982), 63-66.

[J1] B. JiAng, Lectures on Nielsen Fixed Point Theory, Contemp. Math. 14, Amer. Math. Soc., Providence, 1983.

[J2] B. JiAng, Periodic orbits on surfaces via Nielsen fixed point theory, in: TopologyHawaii, K. H. Dovermann (ed.), World Scientific, Singapore, 1992, 101-118.

[J3] B. JIANG, Nielsen theory for periodic orbits and applications to dynamical systems, in: Nielsen Theory and Dynamical Systems, Ch. McCord (ed.), Contemp. Math. 
152, Amer. Math. Soc., Providence, 1993, 183-202; Estimation of the number of periodic orbits, Pacific J. Math. 172 (1996), 151-185.

[J4] B. JiAng, Bounds for fixed points on surfaces, Math. Ann. 311 (1998), 467-479.

[JG] B. JiAng and B. Guo, Fixed points of surface diffeomorphisms, Pacific J. Math. 160 (1993), 67-89.

[JL] B. JiAng and J. Llibre, Minimal sets of periods for torus maps, Discrete Cont. Dynam. Systems 4 (1998), 301-320.

[JW] B. JIANG and S. WANG, Twisted topological invariants associated with representations, in: Topics in Knot Theory, M. E. Bozhüyük (ed.) Kluwer, Dordrecht, 1993, $211-227$.

[K] T.-H. KIAng, The Theory of Fixed Point Classes, Science Press, Beijing, 1979, 1986 (in Chinese); English edition, Springer, Berlin, 1989.

[KL] P. KIRK and C. Livingston, Twisted knot polynomials: inversion, mutation and concordance, Topology 38 (1999), 663-671.

[Ko1] B. Kolev, Entropie topologique et représentation de Burau, C. R. Acad. Sci. Paris 309 (1989), 835-838.

[Ko2] B. Kolev, Point fixe lié à une orbite périodique d'un difféomorphisme de $\mathbb{R}^{2}, \mathrm{C}$. R. Acad. Sci. Paris 310 (1990), 831-833.

[L] S. Lefschetz, Continuous transformations of manifolds, Proc. Nat. Acad. Sci. USA 9 (1923), 90-93; 11 (1925), 290-292; Intersections and transformations of complexes and manifolds, Trans. Amer. Math. Soc. 28 (1926), 1-49.

[Li] X.-S. LIN, Representations of knot groups and twisted Alexander polynomials, preprint, 1990.

[Lo] J. E. Los, Pseudo-Anosov maps and invariant train tracks in the disk: a finite algorithm, Proc. London Math. Soc. 66 (1993), 400-430.

[Ma ] T. MATSUOKA, The number and linking of periodic solutions of periodic systems, Invent. Math. 70 (1983), 319-340; Waveform in dynamical systems of ordinary differential equations, Japan. J. Appl. Math. 1 (1984), 417-434.

[M] S. Moran, The Mathematical Theory of Knots and Braids, An Introduction, NorthHolland Math. Stud. 82, North-Holland, Amsterdam, 1983.

[N1] J. NiELSEN, Über die Minimalzahl der Fixpunkte bei den Abbildungstypen der Ringflächen, Math. Ann. 82 (1921), 83-93; also in: Jakob Nielsen: Collected Mathematical Papers, vol. 1, V. L. Hansen (ed.), Birkhäuser, Boston, 1986, 99-109.

[N2] J. NiELSEN, Untersuchungen zur Topologie des geschlossenen zweiseitigen Flächen, I, Acta Math. 50 (1927), 189-358; English transl.: Investigations in the topology of closed orientable surfaces, I, in: Jakob Nielsen: Collected Mathematical Papers, vol. 1, V. L. Hansen (ed.), Birkhäuser, Boston, 1986, 223-341.

[N3] J. NiELSEN, Fixpunktfrie afbildninger, Mat. Tidsskr. B (1942), 25-41, reviewed by R. Fox, Math. Reviews 7 (1946), 137; English transl.: Fixed point free mappings, in: Jakob Nielsen: Collected Mathematical Papers, vol. 2, V. L. Hansen (ed.), Birkhäuser, Boston, 1986, 221-232.

[R] K. Reidemeister, Automorphismen von Homotopiekettenringen, Math. Ann. 112 (1936), 586-593.

[S] H. Schirmer, A relative Nielsen number, Pacific J. Math. 122 (1986), 459-473.

[T] W. P. Thurston, On the geometry and dynamics of diffeomorphisms of surfaces, Bull. Amer. Math. Soc. 19 (1988), 417-431. 
[Wd] M. WADA, Twisted Alexander polynomial for finitely presentable groups, Topology 33 (1994), 241-256.

[Wa1] S. WANG, Maximum orders of periodic maps on closed surfaces, Topology Appl. 41 (1991), 255-262.

[Wa2] S. WANG, Free degrees of homeomorphisms and periodic maps on closed surfaces, Topology Appl. 46 (1992), 81-87.

[W] F. Wecken, Fixpunktklassen, I, Math. Ann. 117 (1941), 659-671; II, 118 (1942), 216-234; III, 118 (1942), 544-577.

[Y] C. Y. You, A note on periodic points on tori, Beijing Math. 1 (1995), 224-230. 